\title{
Publishing as Social Action: Building Editorial Policies for a Worldwide Journal
}

Serdar M. Değirmencioğlu

\begin{abstract}
Numerous editorial policies and practices are suggested to increase publishing opportunities for scholars in areas of the world where resources are few and English is not the primary language.
\end{abstract}

Keywords. international publishing, cultural imperialism, social action

There is a striking increase in the number of journals in the social sciences. There is also a large number of new journals in psychology and counseling. However, the increase in numbers does not necessarily mean diversity in terms of focus or in terms of editorial policies.

The Journal for Social Action in Counseling and Psychology (JSACP) is a new journal that has distinguished itself in terms of focus right from the start. The journal is intended to provide "a space for examining challenges to the idea that the psychosocial effects of counseling and psychology are generally sufficient, positive, and benign" (Sloan \& Toporek, 2007). This focus heralds a journal whose time has certainly come. 
The journal is grounded in two important and challenging arguments. The founding editors "do not believe that sustaining and expanding the business of counseling and psychology as currently constituted will play a tangible role in the achievement of social justice or the construction of peace and social justice either locally or globally" (Sloan \& Toporek, 2007).

Secondly, they "are concerned that some practices associated with counseling and psychology are worse than ineffective and are, in fact, either part of the problem or do direct harm" (Sloan \& Toporek, 2007).

The journal has been launched with several important questions: "Are psychosocial services actually contributing to the construction of a just society in significant ways? Or are they mostly a distraction that makes helpers feel good as they soothe people who have been more severely wounded by social forces? Are psychological and counseling practices so imbued with cultural biases that they essentially become tools of cultural colonization? Or are they universally applicable techniques that can be empirically validated once and for all?" (Sloan \& Toporek, 2007)

\section{The Challenge}

The questions JSACP is raising and that potential authors are expected to address are certainly not limited to the immediate context within which the journal was founded and where the editors are located. The founding editors of JSACP, among others, are aware of the fact that many counselors and psychologists would readily agree that their work is not limited to a single locale and psychology and counseling are often portrayed as culture-free. More importantly, social action in counseling and psychology is certainly not limited to a given locale (country, region, continent, etc.), to certain ethnic groups, or to specific linguistic lines.

The "Vision and Direction" statements of the journal are certainly not limited to North America, where the majority of counseling and psychology literature is produced. The central to the mission of JSACP is defined as follows: "to promote discourse that is relevant to diverse cultural groups and communities" (Sloan \& Toporek, 2007). The scope of the journal is shaped by "four central intentions":

1. To bring social action research, practice, and training into the forefront of scholarly discourse;

2. To provide a forum for demonstrating effective interventions for addressing social issues;

3. To foster international and intercultural collaboration and understanding;

4. To encourage dialogue between individuals and communities toward the resolution of social problems.

The third intention is clearly a one that defines the geographical scope of the journal widely - so widely that the journal has worldwide coverage. The other three intentions can also be regarded as far-reaching intentions that would require JSACP to be a worldwide ${ }^{1}$ journal.

\footnotetext{
${ }^{1}$ Throughout this paper, "world" is preferred as an inclusive and neutral term. The older concept of "international" assumes that "national" and "nation" carry significant meanings and "nation" and "country" are interchangeable. The fact is "nation" is not the same as country and many countries have more than one nation within itself. The currently popular term "global" is ill-defined and is often used to denote the quick transmission of something (e.g., technology) from one place to another or to many
} 
If the mission of JSACP is defined broadly and if social action that is central to the journal can and does take place all over the world, the challenge is to make the journal a truly international - or a world - journal.

This brief paper suggests that a number of editorial policies need to be put into place and action to make JSACP a more inclusive journal. The challenge is obvious and the task is not easy. The paper is not intended in any sense to be a definitive paper. Rather, the intention is to start a conversation around a number of suggestions and to generate a discussion that can guide the future of the journal.

\section{Social Action Far Away}

The norm in publishing is to fit content into the format of the journal. Journal pages are scarce, tables and figures are costly, and the bibliography needs a page or two to make the paper fit with a given literature. From a social action vantage point, however, a journal should be flexible enough to fit with the content of interest. In other words, a journal devoted to social action should be flexible enough to attract social action work from places that might be quite distant and different.

The following scenario can help the reader consider the challenges involved:

A counselor and a psychologist have been working on police-community tensions, particularly police brutality, in a major city in Greece. They have been working with local youth using an innovative approach. Local youth have been particularly active in documenting and finding ways to reduce police brutality without violent confrontations. Knowing that the problem is common across the country, the counselor and the psychologist suggest to the local youth that they think about preparing a book of cartoons that can help youth in other places deal with the problem. The small book is a major hit across the country and is available on several web sites run by young people. The two activist professionals decide to write about their experience to foster social action among their peers - other professionals. Their work is published in a marginal monthly magazine and attracts some attention from other counselors and psychologists in the country.

The challenge here is obvious: How does this piece of work, written and published in the local language, reach counselors and psychologists who do not speak Greek? The challenge would not be different if the scenario took place in Turkey, Romania, Egypt, Thailand or in any one of the large number of countries that are not located in North America, in Western Europe or in places where the native languages are not linguistically close to dominant languages in counseling and psychology.

The answer to this question requires a full consideration of mechanisms or processes a journal relies on and that JSACP is expected to rely upon. An answer to this question might emerge once the suggestions below are considered.

places at the same time. "Global" often describes the transmission of practices from dominant or powerful centers to the marginal or weak peripheries, and is not synonymous with worldwide, a neutral concept. 


\section{The Editorial Board}

An important starting point for any journal that has a claim to be a world journal is the establishment of a diverse and inclusive editorial board. If the intention is to create a truly diverse editorial board, the board needs to include members from various parts of the world.

The composition of the editorial board is certainly not an obscure issue. Many journals list the names and affiliations of the board members and it is clear that this information carries some significance. The significance and the role of the editorial board, however, are very infrequently discussed in scientific journals (e.g., Gimènez-Toledo, Rodriguez-Garcia \& de la Moneda Corrochano, 2009; Keigher, 2000).

It is even more difficult to find such discussions in journals that claim to be "international". Such journals rarely have explicit policies regarding the composition of the editorial board. It is quite possible to find a journal with a claim to being an "international journal" whose editorial board is composed primarily or exclusively of scholars who reside in a single country (e.g., the US) or in a specific region (e.g., North America). It is even more common for an editorial board to consist only of a single language group.

The problem is by no means limited to counseling or psychology. It is quite easy to identify journals in other fields, even in fields where the social action component is much more salient.

An example might be useful to illustrate he point: The Journal of Human Behavior in the Social Environment is a social work journal that has been published since 1998. The mission of the journal is defined under the "aims and scope" section of the journal web site as follows: The journal "helps social workers firmly grasp developing issues in human behavior theories. It provides an outlet for empirically based articles about human behavior theory that facilitate social workers' practice goals. This innovative journal is the first to address the complexities of human behavior in relation to social work and its relevancy to practice. This makes it an essential resource for classes in human behavior in the social environment. Articles provide you with groundbreaking, up-to-date information on developments in empirically based human behavior theory. They address conceptual and empirical foci which study human behavior as a complex phenomenon. Supported theories target specific behaviors for change, possess clarity by describing in detail the intended change(s), predict the change(s), and facilitate the desired behavioral change(s) through implementation of the model theory."

The aims and scope statement does not limit the journal in any way to a single country. There is nothing in the aims and scope statement that might require the journal to be limited to a single linguistic group. An examination of the editorial board, however, reveals that this journal is intended to be a US journal: The members of the editorial board are listed with their affiliations and abbreviated state names (i.e., state initials such as PA, TX, CT) are included just as they would be listed in a journal that caters only to readers in the US. The board contains a single member from Canada and only a single member outside of North America.

If $J S A C P$ is to "foster international and intercultural collaboration and understanding", or if $J S A C P$ is to serve as a platform for exchange better practices in social action across social,

\footnotetext{
${ }^{2}$ http://0-www.informaworld.com.library.bilgi.edu.tr/smpp/title $\sim \mathrm{db}=\mathrm{all} \sim$ content=t792306870 
cultural and linguistic faultlines, the composition of the editorial board needs to be considered from the very start.

\section{Role for Editorial Board: Averting Editorial Bias}

$J S A C P$ has set out to engage in a difficult task. Agents of social change and those who are engaged in social action often have different priorities. An important component of social action is identifying who has power and which interests are served by any given action, process or tool. An editor cannot claim to be above all kinds of cultural, professional or personal biases. After all, JSACP has explicitly set out to be careful about cultural biases that they essentially become tools of cultural colonization. It is, therefore, necessary to install balancing factors into the publishing process to help the editors steer the journal.

$J S A C P$ is located in the US and is very likely to be influenced to varying degrees by common practices in the US, particularly by the dominant academic culture. Journal editors are expected to be cognizant of and alert to such influences. However, there is no guarantee that an editor can do this alone. Perhaps there is no reason to assume so, provided that an important task for the editorial board is defined as guarding against such influences and averting possible biases. This might be particularly effective if the board cuts across linguistic and academic lines.

\section{Role for Editorial Board: Averting Linguistic Biases}

A particularly important role that the editorial board can play is to challenge the domination of English in psychology and counseling. The status quo is such that English is given the status of royal road to journal pages and this practice is now criticized in various fields (e.g., Ramon, Simonsen, \& Vaiou, 2006). This practice not only puts potential authors whose native language is not English at a clear disadvantage, but also privileges the English spoken in certain countries. A psychologist or counselor whose native language is not English is expected not only to write in English, but write in scholarly English and conform to linguistic "high standards". The royal road is - not surprisingly - not very difficult to navigate for native English-speakers, particularly for those who come from privileged backgrounds, and they are afforded a clear advantage when it comes to having their voices heard in scholarly jounals. English spoken in the US is particularly culture-laden given its openness to new words, grammatical rules, additions from popular culture. Authors who live and work outside of the US often have difficulty comprehending, let alone reproducing peculiarities of English spoken in the US.

Language is neither a neutral space, nor a medium that does not carry cultural biases. Language that is produced and reproduced in an industrial or post-industrial society reflects a very different set of psychological constructs compared to the language that a traditional rural life produces. Given the fact that JSACP is grounded in questions about the dominant modes of practice in pscyhology and counseling and such practices ill-serving populations that are outside of the regions where these dominant practices are produced, JSACP needs to pay particular attention to language.

It is well-known that journal editors as well as practioners in English-speaking countries rarely speak a second language. JSACP has already made a commitment to having Spanishspeaking editors and to including Spanish formally as a medium. The challenge, therefore, is to expand this inclusive move to other languages. This certainly is not an easy task 
particularly if this task is undertaken solely by editors who speak only one or two languages. In fact, it is clear that even a linguistically-savvy editor can only handle several languages.

That is exactly the point where a linguistically diverse editorial board will be very instrumental. If the editorial board is composed of individuals who are competent in a language other than English and the board is designed to include members who speak languages that fit main linguistic lines in the world (e.g., Spanish, French, Portugese, Chinese, Arabic, and so on), JSACP can become a more inclusive journal.

This solution is admittedly one that is intended for breaking the mono-linguistic trend in psychology and counseling. A linguistically diverse editorial board does not address the problem of linguistic inequalities, linguistic domination and the legacy of imperialism in the world. But it is certainly a good start.

It should be noted that language policy is not an easy issue and efforts to engage in multiple-language editing and/or publishing are rare in psychology and counseling. There may be multiple options to consider and different options may be particularly relevant for particular settings.

An example might be well-worth to consider the challenges involved. The Asian Journal of Social Psychology (AJSP) is published by the Asian Association of Social Psychology and the Japanese Group Dynamics Association. It publishes theoretical and empirical papers, as well as book reviews, and "encourages submissions with Asian content and/or Asian authors, but also welcomes high quality submissions from any part of the world". ${ }^{3}$

In keeping with its wide geographical coverage, AJSP maintained a multiple-language submission policy whereby authors could submit their manuscripts in Chinese, Japanese, Korean as well as in English. All articles were published in English to keep the journal in league with other journals in social psychology. The multiple-language submission policy was abandoned on June 15, 2008. The journal now accepts only English-language manuscripts.

\section{Proactive Engagement as Editorial Policy}

This paper suggests that a number of editorial policies need to be put into place and action to make JSACP a more inclusive journal. The challenge is obvious and the task is not easy. To the extent that JSACP departs from mainstream journal practices in psychology and counseling, the editorial process will need to become proactive. In a typical journal, editors play the role of gatekeepers. Very infrequently would they solicit papers to fill in gaps or to highlight a major issue that has not received much attention. A journal on social action would require an editorial approach that is very different from the gatekeeper approach. Put simply, the editorial approach needs to be proactive so that social action may get reported, considered, and disseminated widely.

An example might help to illustrate the point. If psychological concepts that have long served an indigenous community have not been considered in psychology and counseling because they are simply in an indigenous language foreign to English-speakers, proactive editorial engagement might attach special significance to work that provides insight into concepts or cultural practices that are bound to remain neglected by mainstream psychology and counseling.

\footnotetext{
${ }^{3}$ http://www.wiley.com/bw/submit.asp?ref=1367-2223\&site $=1$. 
The scenario mentioned at the beginning of this paper demands a proactive editorial policy as well. For the social action outlined in the scenario to reach JSACP readers, the editorial board needs to include a Greek-speaking member who alerts agents of social action to the possibility of publishing a piece in JSACP. JSACP might be particularly appealing to these activist professionals at least for two reasons: The activist professionals might not be able to publish their work in their own country if the usual professional journals are closed to work focused on social action. Secondly, these activist professionals might be particularly interested in publicizing their work to a larger audience.

\section{Publishing as Social Action}

The suggestions, challenges or tasks that are put forth in this paper might appear to be excessive, too demanding for a new journal, almost overkill. These ideas or tasks need not be too demanding provided that a diverse and inclusive editorial board is at work and primary editors are not expected to speak many languages or to travel all over the world to elicit papers to make the journal a world journal.

A second point that is perhaps a critical to the mission of JSACP is that journal publishing in and of itself is social action. The proactive stance that is recommended in this paper will not appear too demanding if the journal is regarded as a social action process.

The notion of "publishing as social action" is inherent in the mission statements and particularly in the fact that the journal has emerged from two engaged organizations, namely Counselors for Social Justice (CSJ) and Psychologists for Social Responsibility (PsySR).

Put differently, if social action is needed to alter the current publishing practices in mainstream psychology and counseling and a particular type of social action is needed to bring about a truly worldwide journal, such action is particulary significant and valuable. This type of social action can make the journal a sought out publication outlet for counselors and psychologists from all over the world who face major obstacles in having their voices heard.

\section{Editorial Policies for a Worldwide Journal}

This paper suggests that a number of editorial policies need to be put into place and action to make JSACP a more inclusive journal - so inclusive that it can be a journal for social action worldwide. This is obviously a challenge - one that JSACP needs to meet as an innovative social action journal.

If journal publishing is viewed in and of itself as significant social action, JSACP should explicitly address the role and the composition of the editorial board. For JSACP to achieve its social action mission and achieve it with worldwide coverage, JSACP needs to have explicit policies regarding the composition of the editorial board. JSACP (or any other journal) that wants to have worldwide input and influence, the editorial board has to be diverse in terms of gender, ethnicity, location, region, and native language. Explicit policies regarding well-known biases in mainstream psychology will not only make JSACP a more attractive journal, but will also help journal editors avoid biases that are persistent.

Secondly, JSACP needs an explicit policy that requires the editors and the editorial board to be proactive. It is often the case that scholarship in psychology and counseling happens in sterile settings, such as the clinic, the classroom, the lab, or generic conference rooms. If social action is to reported in JSACP, a diverse editorial board has to seek and invite work 
that involves social action around the worldwide. This type of proactive collective social action on the part of the editorial board can make JSACP a sought after publication outlet for counselors and psychologists from all over the world who face major obstacles in having their voices heard.

The main argument here is that in order to "foster international and intercultural collaboration and understanding" and to serve as a platform for exchange better practices in social action across social, cultural and linguistic faultlines, JSACP has to build and follow explicit editorial policies that fit with its goals.

The suggestions in this paper are not intended in any sense to be definitive suggestions. Rather, the intention is to start a conversation around "journal publishing as social action" and "proactive" editorial policies that can help JSACP engage in the task of "journal publishing as social action".

Once a consensus emerges around ways of engaging in publishing a "journal as social action", then this consensus should be formalized and publicized as journal editorial policy. This overt commitment would better attract counselors and psychologists engaged in social action to the journal.

Contact information/Correspondence:

Serdar M. Değirmencioğlu

Cumhuriyet University, Turkey

e-mail: serdardegirmencioglu@gmail.com

\section{References}

Gimènez-Toledo, E., Rodriguez-Garcia, G. \& de la Moneda Corrochano, M. (2009). Spanish scientific journals on Psychology (II): Editorial quality, visibility, internationality and editors attitude toward Open Access. Psychology Science Quarterly, 51 (Supplement), 119-134.

Keigher, S. M. (2000). Knowledge development in Health \& Social Work. Health \& Social Work, 25(1), 3-8.

Ramon, M. D. G., Simonsen, K. \& Vaiou, D. (2006) Guest Editorial: Does Anglophone hegemony permeate Gender, Place and Culture? Gender, Place \& Culture, 13 (1),1-5.

Sloan, T. \& Toporek, R. L. (2007). On social action in counseling and psychology: Visions for the journal. Journal for Social Action in Counseling and Psychology, 1 (1). 